\title{
Electronic states and nature of bonding of the molecule NiGe by all electron ab initio Hartree-Fock (HF) and configuration interaction (Cl) calculations and mass spectrometric equilibrium experiments
}

Shim, Irene; Kingcade, Joseph E.; Gingerich, Karl A.

Published in:

Journal of Chemical Physics

Link to article, DOI:

10.1063/1.454967

Publication date:

1988

Document Version

Publisher's PDF, also known as Version of record

Link back to DTU Orbit

Citation (APA):

Shim, I., Kingcade, J. E., \& Gingerich, K. A. (1988). Electronic states and nature of bonding of the molecule $\mathrm{NiGe}$ by all electron ab initio Hartree-Fock (HF) and configuration interaction (Cl) calculations and mass spectrometric equilibrium experiments. Journal of Chemical Physics, 89(5), 3104-3112.

https://doi.org/10.1063/1.454967

\section{General rights}

Copyright and moral rights for the publications made accessible in the public portal are retained by the authors and/or other copyright owners and it is a condition of accessing publications that users recognise and abide by the legal requirements associated with these rights.

- Users may download and print one copy of any publication from the public portal for the purpose of private study or research.

- You may not further distribute the material or use it for any profit-making activity or commercial gain

- You may freely distribute the URL identifying the publication in the public portal 


\title{
Electronic states and nature of bonding of the molecule NiGe by all electron $a b$ initio Hartree-Fock (HF) and configuration interaction (Cl) calculations and mass spectrometric equilibrium experiments
}

\author{
Irene Shim \\ Chemical Physics, Chemistry Department B, Technical University of Denmark, DTH 301, DK-2800 \\ Lyngby, Denmark
}

Joseph E. Kingcade, Jr. ${ }^{\text {a) }}$ and Karl A. Gingerich

Department of Chemistry, Texas A\&M University, College Station, Texas 77843

(Received 29 February 1988; accepted 26 May 1988)

\begin{abstract}
All electron $a b$ initio Hartree-Fock (HF) and configuration interaction (CI) calculations have been applied to investigate the low-lying electronic states of the NiGe molecule. The ground state of the NiGe molecule is predicted to be ${ }^{1} \Sigma^{+}$. The chemical bond in the ${ }^{1} \Sigma^{+}$ ground state is a double bond composed of one $\sigma$ and one $\pi$ bond. The $\sigma$ bond is due to a delocalized molecular orbital formed by combining the $\mathrm{Ni} 4 s$ and the Ge $4 p \sigma$ orbitals. The $\pi$ bond is a partly delocalized valence bond, originating from the coupling of the $3 d \pi$ hole on $\mathrm{Ni}$ with the $4 p \pi$ electron on Ge. The low-lying electronic states of the NiGe molecule have all been characterized by the symmetry of the hole in the $3 d$ shell of Ni. The dissociation energy of the NiGe molecule has been determined from our high temperature mass spectrometric equilibrium data in combination with the theoretical results as $D_{0}^{\circ}=286.8 \pm 10.9 \mathrm{~kJ} \mathrm{~mol}^{-1}$. The standard heat of formation of the NiGe molecule has been obtained as $\Delta H_{f, 298}^{\circ}$ $=514 \pm 12 \mathrm{~kJ} \mathrm{~mol}^{-1}$.
\end{abstract}

\section{INTRODUCTION}

Investigations of the physical and chemical properties of small transition metal containing molecules are of considerable current interest. ${ }^{1-4}$ In continuation of our recent published work elucidating the electronic structure and thermodynamic properties of the first platinum metal triad mono carbides ${ }^{5-8}$ and the molecules $\mathrm{PdGe}^{9}$ and $\mathrm{PdSi}^{10}$ we are reporting in the present paper the investigations of the gaseous molecule NiGe.

By studying the interaction between the Ni atom and the Ge atom we are able to explore the changes occurring in the electronic structure and nature of bonding due to the exchange of the Pd atom by the $\mathrm{Ni}$ atom. Furthermore, it is interesting to compare the chemical bond in the heteronuclear $\mathrm{NiGe}$ molecule as described in our present theoretical work with our previous results concerning the homonuclear molecules $\mathrm{Ni}_{2}{ }^{3,11}$ and $\mathrm{Ge}_{2}{ }^{12,13}$

The NiGe molecule has not previously been investigated theoretically, and the experimental work concerning this molecule is limited to brief reports on the measurement of its dissociation energy. ${ }^{14,15}$

In the present work the $\mathrm{NiGe}$ molecule has been investigated by all electron $a b$ initio Hartree-Fock (HF) and configuration interaction (CI) calculations and also by high temperature mass spectrometric measurements. The electronic structure and the nature of bonding of the NiGe molecule have been revealed by the theoretical work, and the results obtained have been utilized to derive the dissociation energy of the molecule from the experimental mass spectrometric equilibrium data.

\footnotetext{
a) Permanent address: Division of Natural Science, Blinn College, Brenham, Texas 77833 .
}

The HF calculations for the NiGe molecule have been carried out in the Hartree-Fock-Roothaan formalism. ${ }^{16}$ The integrals have been calculated using the program MOLECULE. ${ }^{17}$ For the HF calculations we have utilized the ALCHEMY program system, ${ }^{18}$ and the CI calculations have been performed using ALCHEMY in conjunction with the program ENERGY ${ }^{19}$ for generating the symbolic energy expressions. Finally, the deformation density map has been produced by a program especially designed for contour plotting of orbitals, densities, and electrostatic potentials. ${ }^{20}$

\section{THEORETICAL INVESTIGATIONS}

\section{A. Basis sets and atomic calculations}

The basis sets consisted of Gaussian type functions. For the $\mathrm{Ni}$ atom we have employed the basis set previously used in our work on the $\mathrm{Ni}_{2}$ molecule. ${ }^{3}$ It is essentially Wachters' basis set, ${ }^{21}$ but it has been modified by contracting the two most diffuse $s$ functions and by the addition of two $p$ functions with exponents 0.27 and 0.10 , and also a diffuse $d$ function with exponent 0.1557 .

The basis set for the Ge atom has previously been used in our work on the $\mathrm{Ge}_{2}$ molecule. ${ }^{12,13}$ It was obtained by contracting slightly the most diffuse $s$ and $p$ functions in Huzinaga's basis ${ }^{22}$ and by adding an extra $d$ function. For both atoms, $\mathrm{Ni}$ and $\mathrm{Ge}$, the primitive basis sets $(14 s, 11 p, 6 d)$ have been contracted to $(8 s, 6 p, 3 d)$ using a segmented contraction scheme.

In Table I, we compare the relative energies of the lowlying terms of the $\mathrm{Ni}$ and $\mathrm{Ge}$ atoms as derived in the $\mathrm{HF}$ calculations with the corresponding experimental data. It is noted that although the calculated splittings for both atoms are somewhat larger than the experimental values, the se- 
TABLE I. Relative energies (in a.u.) of the lowest-lying terms of the Ni and the Ge atoms. The calculated energies are results of HF calculations.

\begin{tabular}{ccrc}
\hline \hline Atom & Term & Calculated & Experimental $^{\mathrm{a}}$ \\
\hline $\mathrm{Ni}$ & ${ }^{3} D(3 d)^{9}(4 s)^{1}$ & 0.000000 & 0.000000 \\
$\mathrm{Ni}$ & ${ }^{3} F(3 d)^{8}(4 s)^{2}$ & -0.047022 & 0.001095 \\
$\mathrm{Ni}$ & ${ }^{1} D(3 d)^{9}(4 s)^{1}$ & 0.014609 & 0.012204 \\
$\mathrm{Ni}$ & ${ }^{1} D(3 d)^{8}(4 s)^{2}$ & 0.025979 & 0.058275 \\
$\mathrm{Ni}$ & ${ }^{1} S(3 d)^{10}$ & 0.151990 & 0.063776 \\
$\mathrm{Ge}$ & ${ }^{3} P(4 s)^{2}(4 p)^{2}$ & 0.000000 & 0.000000 \\
$\mathrm{Ge}$ & ${ }^{1} D(4 s)^{2}(4 p)^{2}$ & 0.043006 & 0.028050 \\
$\mathrm{Ge}$ & ${ }^{1} S(4 s)^{2}(4 p)^{2}$ & 0.106941 & 0.070159 \\
\hline \hline
\end{tabular}

"Center of gravity of each multiplet has been derived from data of C. E. Moore, Natl. Bur. Stand. Circ. No. 467 (U.S. GPO, Washington, D.C., 1952), Vol. 2.

quences of the terms originating from a single orbital configuration are correct. For the $\mathrm{Ni}$ atom the calculated splitting between the ${ }^{3} D(3 d)^{9}(4 s)^{1}$ and the ${ }^{3} F(3 d)^{8}(4 s)^{2}$ terms reveals the well-known fact ${ }^{23}$ that the correlation energy differs by $\sim 0.05$ a.u. in the configurations $(3 d)^{9}(4 s)^{1}$ and $(3 d)^{8}(4 s)^{2}$. This fact should always be kept in mind when performing calculations on molecules containing the $\mathrm{Ni}$ atom, and only by performing large $\mathrm{CI}$ calculations is it possible to account simultaneously for the atomic and the molecular correlation.

\section{B. HF calculations on selected electronic states of the NiGe molecule}

As is usual for a coordinatively unsaturated transition metal containing molecule, it is very difficult to make a qualified guess as to the space and spin symmetries of the electronic ground state for the NiGe molecule. Since the ${ }^{3} D(3 d)^{9}(4 s)^{1}$ and the ${ }^{3} F(3 d)^{8}(4 s)^{2}$ terms of Ni are almost degenerate, the ground state of the NiGe molecule should be sought among the states arising by coupling of the angular momenta of the above mentioned terms with those of the ${ }^{3} P(4 s)^{2}(4 p)^{2}$ ground term of the Ge atom. However, this procedure gives rise to numerous singlet, triplet, and quintet states of various space symmetries, and therefore it hardly offers an appropriate guidance for determining the symmetry of the electronic ground state of the NiGe molecule.

Finally we decided to consider the $\mathrm{Ni}$ atom in either of the configurations $(3 d)^{9}(4 s)^{1}$ or $(3 d)^{8}(4 s)^{2}$ as having holes in $3 d$ subshells of the specific symmetries, i.e., $3 d \delta, 3 d \pi$, or $3 d \sigma$, and we then investigated the interaction between such "prepared" $\mathrm{Ni}$ atoms and the Ge atom with either of the valence shell configurations $(4 p \pi)^{2},(4 p \pi)^{1}(4 p \sigma)^{1}$, or $(4 p \sigma)^{2}$ by carrying out HF calculations.

In Table II, we present some of the results obtained in the HF calculations performed on the NiGe molecule at the internuclear distance 4.60 a.u. Table II shows the calculated energies of the various states as well as their valence shell configurations. Also included are the total number of $d$ electrons and the gross atomic charge associated with the $\mathrm{Ni}$ atom. It is noted that states originating from both the $(3 d)^{8}$ and the $(3 d)^{9}$ configurations of $\mathrm{Ni}$ are present, but only states arising from the $(3 d)^{9}$ configuration of the $\mathrm{Ni}$ atom are found to be bound relative to the free atoms. The results for the lowest lying ${ }^{1} \Sigma^{+}$state have been added retrospectively, because the configuration $(2 \delta)^{4}(6 \pi)^{3}(7 \pi)^{1}(12 \sigma)^{2}(13 \sigma)^{2}(14 \sigma)^{2}$ constitutes the major configuration in the final $\mathrm{CI}$ wave function.

Analyses of the wave functions obtained in the HF calculations indeed reveal that most of the states in Table II can be characterized as having localized holes in the $3 d$ subshells of $\mathrm{Ni}$ of specific symmetries.

Thus, the ${ }^{5} \Delta,{ }^{3} \Pi, \Phi$, and ${ }^{3} \Delta$ states in Table II all have a localized hole in the $3 d \delta$ subshell of $\mathrm{Ni}$, and they represent the interaction between a $\mathrm{Ni}$ atom with a hole in the $3 d \delta$ subshell and $\mathrm{a}$ Ge atom with the valence shell configurations $(4 p \pi)^{2},(4 p \pi)^{1}(4 p \sigma)^{1}$, and $(4 p \sigma)^{2}$, respectively. Likewise, the ${ }^{5} \Sigma^{-}$state and the most stable ${ }^{3} \Pi$ state in Table II both have a hole in the $3 d \sigma$ subshell of the $\mathrm{Ni}$ atom, and they represent the interactions between a $\mathrm{Ni}$ atom with a hole in the $3 d \sigma$ subshell and a Ge atom with the valence shell config-

TABLE II. Total energies for the NiGe molecule as resulting from HF treatments at the internuclear distance 4.60 a.u. Also included are the gross atomic charge and the total number of $d$ electrons on the $\mathrm{Ni}$ atom.

\begin{tabular}{|c|c|c|c|c|c|c|c|c|c|c|}
\hline \multirow[b]{2}{*}{ State } & \multicolumn{7}{|c|}{$\begin{array}{l}\text { Valence shell } \\
\text { configuration }\end{array}$} & \multirow{2}{*}{$\begin{array}{c}\text { Energy }^{a} \\
\text { (a.u.) }\end{array}$} & \multirow{2}{*}{$\begin{array}{c}\text { Number of } \\
d \text { electrons } \\
\text { on } \mathrm{Ni}\end{array}$} & \multirow{2}{*}{$\begin{array}{c}\text { Gross } \\
\text { atomic } \\
\text { charge } \\
\text { on } \mathrm{Ni}\end{array}$} \\
\hline & $2 \delta$ & $6 \pi$ & $7 \pi$ & $12 \sigma$ & $13 \sigma$ & $14 \sigma$ & $15 \sigma$ & & & \\
\hline${ }^{5} \Delta$ & 3 & 4 & 2 & 2 & 2 & 1 & 0 & -0.042781 & 8.98 & 0.22 \\
\hline${ }^{5} \Sigma^{-}$ & 4 & 4 & 2 & 2 & 1 & 1 & 0 & -0.037963 & 9.01 & 0.29 \\
\hline${ }^{3} \Pi, \Phi^{\mathbf{b}}$ & 3 & 4 & 1 & 2 & 2 & 2 & 0 & -0.029992 & 8.94 & -0.08 \\
\hline${ }^{1} \Sigma^{+}$ & 4 & 3 & 1 & 2 & 2 & 2 & 0 & -0.016613 & 8.97 & -0.02 \\
\hline${ }^{3} \Pi$ & 4 & 4 & 1 & 2 & 2 & 1 & 0 & -0.007091 & 8.98 & 0.00 \\
\hline${ }^{5} \Sigma^{+}$ & 2 & 4 & 2 & 2 & 2 & 2 & 0 & 0.001975 & 8.01 & 0.09 \\
\hline${ }^{5} \Pi, \Phi^{b}$ & 3 & 4 & 1 & 2 & 2 & 1 & 1 & 0.012229 & 8.92 & -0.16 \\
\hline${ }^{7} \mathbf{\Sigma}^{+}$ & 2 & 4 & 2 & 2 & 2 & 1 & 1 & 0.019480 & 8.02 & 0.25 \\
\hline${ }^{5} \Pi$ & 2 & 4 & 1 & 2 & 2 & 2 & 1 & 0.027583 & 7.99 & -0.04 \\
\hline${ }^{3} \Delta$ & 3 & 4 & 0 & 2 & 2 & 2 & 1 & 0.039999 & 8.86 & -0.35 \\
\hline${ }^{3} \Pi$ & 4 & 3 & 0 & 2 & 2 & 2 & 1 & 0.047095 & 8.90 & -0.33 \\
\hline${ }^{3} \Sigma^{-}$ & 4 & 4 & 2 & 2 & 2 & 0 & 0 & 0.069733 & 8.18 & 0.21 \\
\hline${ }^{1} \Sigma^{+}$ & 4 & 4 & 0 & 2 & 2 & 2 & 0 & 0.131499 & 9.60 & -0.25 \\
\hline
\end{tabular}

Energy of NiGe minus energy of the ${ }^{3} D(3 d)^{9}(4 s)^{1} \mathrm{Ni}$ and the ${ }^{3} P(4 s)^{2}(4 p)^{2} \mathrm{Ge}$ atoms.

${ }^{b}$ The wave function represents a mixture of orbital angular momenta. 
urations $(4 p \pi)^{2}$ and $(4 p \pi)^{1}(4 p \sigma)^{1}$. The higher lying ${ }^{3} \Pi$ state and the lowest lying ${ }^{1} \Sigma^{+}$state arise from a $\mathrm{Ni}$ atom with a hole in the $3 d \pi$ subshell interacting with the Ge atom with the valence configurations $(4 p \sigma)^{2}$ and $(4 p \pi)^{1}(4 p \sigma)^{1}$, respectively.

The HF calculations mainly serve to determine a set of molecular orbitals suitable for use in the $\mathrm{CI}$ calculations. For this purpose we have included HF calculations of the ${ }^{5} \Pi, \Phi$ mixture of states, since this state arises from the ${ }^{5} \Delta$ state by promoting one electron from the partly filled $\pi$ orbital into an empty $\sigma$ orbital. This accomplishes that a virtual $\sigma$ orbital is changed into an occupied one.

\section{Results of $\mathrm{Cl}$ calculations on the NiGe molecule}

We have performed $\mathrm{CI}$ calculations on the NiGe molecule by allowing full reorganization within the valence shells of the molecule, i.e., within the orbital set $2 \delta, 6 \pi, 7 \pi, 12 \sigma$, $13 \sigma, 14 \sigma$, and $15 \sigma$. This ensures that the wave functions are appropriate for describing the molecule in the dissociation limit. The calculations have been carried out in the subgroup $C_{2 v}$ of the full symmetry group $C_{\infty v}$ of the NiGe molecule. The number of configurations included within each symmetry species of $C_{2 v}$ reaches 60 for the septet states, 590 for the quintet states, 1746 for the triplet states, and 1308 for the singlet states.

In the present work we have aimed at using just a single set of molecular orbitals to describe all the low-lying electronic states of the $\mathrm{NiGe}$ molecule in the $\mathrm{CI}$ calculations. In order to identify an appropriate set of molecular orbitals we have carried out the above-described $\mathrm{CI}$ calculations at the internuclear distance 4.60 a.u. utilizing the molecular orbitals optimized for some of the states presented in Table II, namely ${ }^{5} \Delta,{ }^{3} \Pi, \Phi,{ }^{5} \Sigma^{-},{ }^{5} \Pi, \Phi,{ }^{5} \Sigma^{+},{ }^{7} \Sigma^{+},{ }^{5} \Pi$, as well as the lowest lying ${ }^{1} \Sigma^{+}$state. As expected, the interaction between the ${ }^{3} D(3 d)^{9}(4 s)^{1} \mathrm{Ni}$ and the ${ }^{3} P(4 s)^{2}(4 p)^{2} \mathrm{Ge}$ is described in the CI calculations based on the molecular orbitals optimized for the states ${ }^{5} \Delta,{ }^{3} \Pi, \Phi,{ }^{1} \Sigma^{+},{ }^{5} \Sigma^{-},{ }^{5} \Pi, \Phi$, whereas the CI calculations based on the molecular orbitals optimized for the states ${ }^{5} \Sigma^{+},{ }^{7} \Sigma^{+}$, and ${ }^{5} \Pi$ are describing the interaction between the ${ }^{3} F(3 d)^{8}(4 s)^{2} \mathrm{Ni}$ and the ${ }^{3} P(4 s)^{2}(4 p)^{2} \mathrm{Ge}$ atoms. The preliminary calculations revealed that the $(3 d)^{9}(4 s)^{1}$ configuration of $\mathrm{Ni}$ led to the formation of the most stable NiGe molecule. In the present investigation it has not been our intention to perform the extensive CI calculations required to account for both the atomic and the molecular correlation, and therefore we have chosen to report in detail only the interactions between the $\mathrm{Ni}$ and $\mathrm{Ge}$ atoms that involve the $(3 d)^{9}(4 s)^{1}$ configuration of $\mathrm{Ni}$.

The lowest total energy of the NiGe molecule formed when the $\mathrm{Ni}$ atom in the $(3 d)^{9}(4 s)^{1}$ is interacting with the Ge atom in the $(4 s)^{2}(4 p)^{2}$ configuration was observed in the CI calculations based on the molecular orbitals optimized for the ${ }^{5} \Pi, \Phi$ state of Table II. Therefore, we are presenting the CI results based on these molecular orbitals below.

The molecular orbitals have been optimized for the ${ }^{5} \Pi, \Phi$ mixture of states at the internuclear distances 3.6 , $4.2,4.6,5.0,5.5,6.2$, and 12.0 a.u., and the resulting orbitals have been utilized to perform the CI calculations described above. Over the entire range of internuclear distances considered the $\mathrm{Ni} 3 d \sigma$ orbital is essentially contained in the molecular orbitals $12 \sigma$ and $13 \sigma$. Since the orbitals $12 \sigma$ and $13 \sigma$ are fully occupied in the configuration $(2 \delta)^{3}(6 \pi)^{4}(7 \pi)^{1}(12 \sigma)^{2}(13 \sigma)^{2}(14 \sigma)^{1}(15 \sigma)^{1}$, the $(3 d)^{9}(4 s)^{1}$ configuration of the $\mathrm{Ni}$ atom is essentially retained in the SCF calculations on the mixed ${ }^{5} \Pi, \Phi$ state. Therefore the limited CI calculations performed in the present investigation describe the interaction between the $\mathrm{Ni}$ and the $\mathrm{Ge}$ atoms in the configurations $(3 d)^{9}(4 s)^{1}$ and $(4 s)^{2}(4 p)^{2}$, respectively, at all the internuclear distances considered.

The potential energies obtained in the CI calculations have been fitted to Morse curves, and in Fig. 1 we present the potential energy curves for 17 low-lying electronic states of the NiGe molecule. The equilibrium distances, the vibrational frequencies, and the transition energies derived in the fitting process are reported in Table III. Also included is a lower bound to the dissociation energy for the ${ }^{1} \Sigma^{+}$ground state, which has been determined at the difference between the energies of the NiGe molecule at the equilibrium distance and at the internuclear distance 12.0 a.u.

\section{The ${ }^{1} \Sigma^{+}$ground state of the NGGe molecule}

Table IV shows the Mulliken population analyses of the ${ }^{1} \Sigma^{+}$ground state of the NiGe molecule as described in the

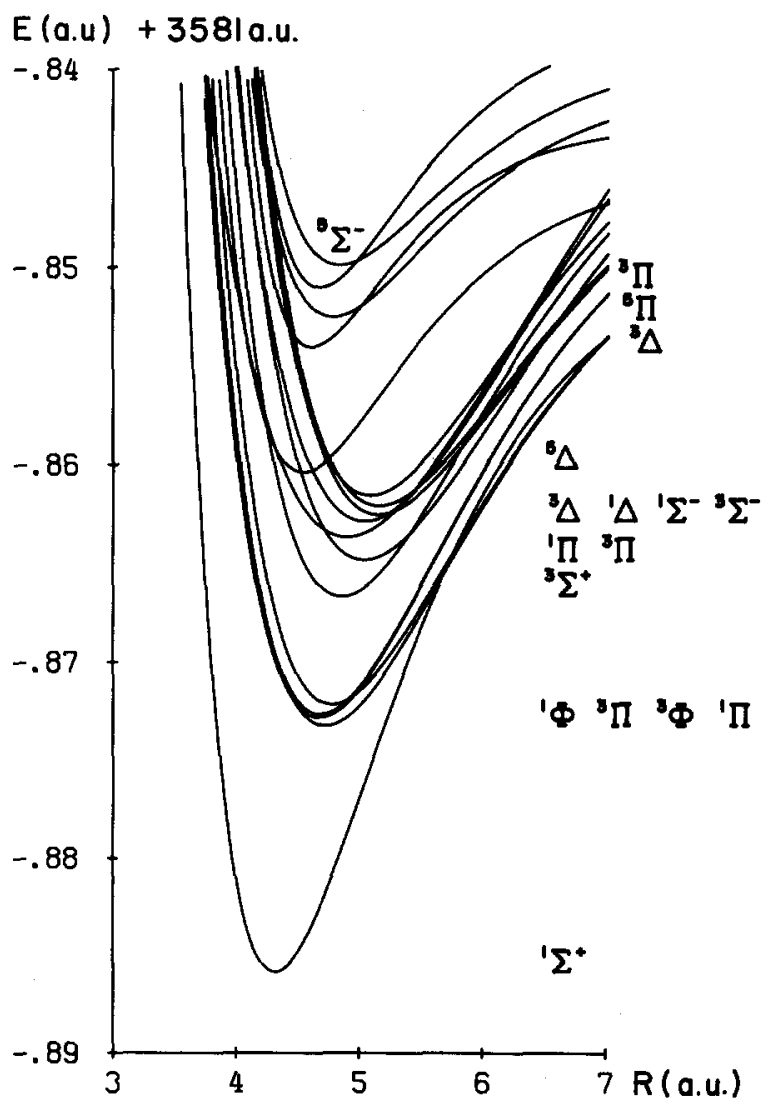

FIG. 1. Potential energy curves for 17 low-lying electronic states of the $\mathrm{NiGe}$ molecule as derived in the CI calculations. The states are labeled according to the increasing transition energies shown in Table III. 
TABLE III. Spectroscopic constants for the low-lying electronic states of the NiGe molecule as derived in Cl calculations.

\begin{tabular}{lcccc}
\hline \hline $\begin{array}{c}\text { State distance (a.u.) } \\
\text { Equilibrium }\end{array}$ & $\begin{array}{c}\text { Transition } \\
\text { energy (eV) }\end{array}$ & $\begin{array}{c}\text { Vibrational } \\
\text { frequency }\left(\mathrm{cm}^{-1}\right)\end{array}$ & $\begin{array}{c}\text { Dissociation } \\
\text { energy (eV) }\end{array}$ \\
\hline${ }^{1} \Sigma^{+}$ & 4.31 & 0.00 & 239 & 1.21 \\
${ }^{1} \Phi$ & 4.73 & 0.34 & 164 & \\
${ }^{3} \Pi$ & 4.68 & 0.35 & 170 & \\
${ }^{3} \Phi$ & 4.69 & 0.36 & 170 & \\
${ }^{1} \Pi$ & 4.78 & 0.37 & 160 & \\
${ }^{3} \Sigma^{+}$ & 4.86 & 0.52 & 157 & \\
${ }^{1} \Pi$ & 5.04 & 0.57 & 141 & \\
${ }^{3} \Pi$ & 4.90 & 0.60 & 129 & \\
${ }^{3} \Delta$ & 5.04 & 0.62 & 137 & \\
${ }^{3} \Delta$ & 5.16 & 0.63 & 137 & \\
${ }^{1} \Sigma^{-}$ & 5.16 & 0.65 & 136 & \\
${ }^{3} \Sigma^{-}$ & 5.09 & 0.66 & 142 & \\
${ }^{5} \Delta$ & 4.55 & 0.69 & 177 & \\
${ }^{3} \Delta$ & 4.62 & 0.86 & 192 & \\
${ }^{5} \Pi$ & 4.79 & 0.91 & 149 & \\
${ }^{3} \Pi$ & 4.69 & 0.95 & 186 & \\
${ }^{3} \Sigma^{-}$ & 4.85 & 0.98 & 141 & \\
\hline \hline
\end{tabular}

- Derived as the difference between the total molecular energies at the equilibrium distance and at the internuclear distance 12 a.u.

$\mathrm{HF}$ and in the CI calculations at the internuclear distance 4.60 a.u. It is noted that the population analyses of the HF and the $\mathrm{CI}$ wave functions resemble each other closely. This reflects the fact that the configuration $(2 \delta)^{4}(6 \pi)^{3}(7 \pi)^{1}(12 \sigma)^{2}(13 \sigma)^{2}(14 \sigma)^{2}$ contributes $\sim 73 \%$ to the wave function at the internuclear distance $4.60 \mathrm{a} . \mathrm{u}$. In addition, it is noted that the populations of the $\mathrm{Ni} 3 d$ and $4 s$ orbitals in the ' $\boldsymbol{\Sigma}^{+}$ground state of the NiGe molecule are very much like those of the $\mathrm{Ni}^{3} \mathrm{D}(3 d)^{9}(4 s)^{1}$ ground term atom.

The chemical bond in the ' $\Sigma$ ' ground state of the NiGe molecule is a double bond consisting of one $\sigma$ and one $\pi$ bond. The $\sigma$ bond is mainly due to the delocalized molecular orbital composed of the $4 s \sigma$ orbital of $\mathrm{Ni}$ and the $4 p \sigma$ orbital of $\mathrm{Ge}$. The $\pi$ bond essentially originates from the valence bond coupling of the localized hole in the $3 d \pi$ subshell of $\mathrm{Ni}$ and the singly occupied $4 p \pi$ orbital of Ge.

Our interpretation of the chemical bond in the NiGe molecule is supported by the results shown in Tables $\mathrm{V}$ and VI. Table V shows the contributions of the major configurations to the ${ }^{1} \Sigma^{+}$ground state of the NiGe molecule, and Table VI shows the populations in the natural valence orbitals as functions of the internuclear distance.

It is noted that the first configuration in Table $\mathrm{V}$ is the closed shell ${ }^{1} \Sigma^{+}$state of Table II. This configuration, however, only contributes significantly to the wave function at internuclear distances shorter than the equilibrium distance of the molecule. Thus, even at the internuclear distance 4.20 a.u. that is 0.11 a.u. shorter than the equilibrium distance of the molecule, it only makes a contribution of $20 \%$. Over the whole range of internuclear distances shown in Table $\mathrm{V}$, the fourth configuration $(2 \delta)^{4}(6 \pi)^{3}(7 \pi)^{1}(12 \sigma)^{2}(13 \sigma)^{2}(14 \sigma)^{2}$ makes the largest contribution to the wave function of the ${ }^{1} \Sigma^{+}$ground state. It is noted that this configuration is essential for our interpretation of the chemical bond as arising from a delocalized $\sigma$ orbital $(14 \sigma)$, and a valence bond coupling between the $3 d \pi$ hole of $\mathrm{Ni}(6 \pi)$ and the $4 p \pi$ electron of $\mathrm{Ge}(7 \pi)$. The admixture of the configurations 5 and 6 introduces left-right correlation into the $\sigma$ bond, while configuration 1 causes some delocalization of the valence bond coupled $\pi$ bond.

From Table VI it is recognized that the $4 s$ orbital of $\mathrm{Ge}$ as well as the $3 d \sigma$ and $3 d \delta$ orbitals of $\mathrm{Ni}$ are fully occupied over the whole range of internuclear distances shown. The $14 \sigma$ and $15 \sigma$ orbitals turn into the localized orbitals, i.e., $4 s$ on $\mathrm{Ni}$ and $4 p \sigma$ on $\mathrm{Ge}$ as the dissociation limit is approached. Likewise, the populations shown in Table VI supports the presence of the localized hole in the $3 d \pi$ orbital of $\mathrm{Ni}$ and the localized electron in the $4 p \pi$ orbital of Ge.

TABLE IV. Mulliken population analyses of the valence orbitals of the NiGe molecule in the ${ }^{~} \Sigma^{+}$ground state. The wave functions have been derived in HF and CI calculations at the internuclear distance 4.60 a.u.

\begin{tabular}{|c|c|c|c|c|c|c|c|c|c|c|c|}
\hline \multirow[b]{3}{*}{ Method } & \multirow[b]{3}{*}{ Orbital } & \multirow{2}{*}{\multicolumn{2}{|c|}{$\begin{array}{c}\text { Atomic } \\
\text { population }\end{array}$}} & \multirow{3}{*}{$\begin{array}{c}\text { Overlap } \\
\text { population }\end{array}$} & \multicolumn{6}{|c|}{ Orbital analyses } & \multirow{3}{*}{$\begin{array}{c}\text { Occupation } \\
\text { number }\end{array}$} \\
\hline & & & & & \multicolumn{3}{|c|}{$\mathbf{N i}$} & \multicolumn{3}{|c|}{$\mathrm{Ge}$} & \\
\hline & & $\mathrm{Ni}$ & $\mathbf{G e}$ & & $s$ & $p$ & $d$ & $s$ & $p$ & $d$ & \\
\hline \multirow[t]{6}{*}{$\mathbf{H F}$} & $12 \sigma$ & 1.74 & 0.28 & -0.03 & 0.03 & 0.00 & 1.70 & 0.25 & 0.02 & 0.00 & 2 \\
\hline & $13 \sigma$ & 0.25 & 1.49 & 0.26 & 0.08 & 0.04 & 0.26 & 1.61 & 0.01 & 0.00 & 2 \\
\hline & $14 \sigma$ & 0.81 & 1.07 & 0.11 & 0.82 & 0.04 & 0.01 & 0.07 & 1.05 & 0.01 & 2 \\
\hline & $6 \pi$ & 3.01 & 0.00 & -0.01 & 0.00 & 0.00 & 3.00 & 0.00 & 0.00 & 0.00 & 3 \\
\hline & $7 \pi$ & 0.01 & 0.93 & 0.05 & 0.00 & 0.04 & 0.00 & 0.00 & 0.96 & 0.00 & 1 \\
\hline & $2 \delta$ & 4.00 & 0.00 & 0.00 & 0.00 & 0.00 & 4.00 & 0.00 & 0.00 & 0.00 & 4 \\
\hline Total & & 27.82 & 31.78 & 0.40 & 6.93 & 12.13 & 8.97 & 7.91 & 14.04 & 10.02 & \\
\hline \multirow[t]{7}{*}{ CI } & $12 \sigma$ & 1.86 & 0.12 & 0.01 & 0.03 & 0.00 & 1.84 & 0.12 & 0.01 & 0.01 & 1.99 \\
\hline & $13 \sigma$ & 0.15 & 1.64 & 0.18 & 0.15 & 0.02 & 0.07 & 1.73 & 0.01 & 0.00 & 1.98 \\
\hline & $14 \sigma$ & 0.80 & 0.91 & 0.15 & 0.85 & 0.00 & 0.02 & 0.06 & 0.92 & 0.00 & 1.87 \\
\hline & $15 \sigma$ & 0.11 & 0.08 & -0.04 & 0.05 & 0.03 & 0.00 & 0.00 & 0.05 & 0.00 & 0.14 \\
\hline & $6 \pi$ & 2.79 & 0.35 & 0.18 & 0.00 & 0.02 & 2.85 & 0.00 & 0.43 & 0.01 & 3.32 \\
\hline & $7 \pi$ & 0.12 & 0.60 & 0.00 & 0.00 & 0.02 & 0.10 & 0.00 & 0.60 & 0.00 & 0.72 \\
\hline & $2 \delta$ & 3.99 & 0.00 & 0.00 & 0.00 & 0.00 & 3.99 & 0.00 & 0.00 & 0.00 & 3.99 \\
\hline Total & & 27.82 & 31.70 & 0.48 & 7.08 & 12.10 & 8.88 & 7.91 & 14.01 & 10.02 & \\
\hline
\end{tabular}


TABLE V. The major contributions to the wave functions describing the ' $\Sigma^{+}$ground state of the NiGe molecule as functions of the internuclear distance.

\begin{tabular}{|c|c|c|c|c|c|c|c|c|c|c|c|c|c|}
\hline \multirow{2}{*}{\multicolumn{7}{|c|}{ Valence shell configuration }} & \multicolumn{7}{|c|}{ Contribution of the valence shell configuration (\%) } \\
\hline & & & & & & & & & Intern & ar dista & (a.u.) & & \\
\hline $12 \sigma$ & $13 \sigma$ & $14 \sigma$ & $15 \sigma$ & $6 \pi$ & $7 \pi$ & $2 \delta$ & 3.6 & 4.2 & 4.6 & 5.0 & 5.5 & 6.2 & 12.0 \\
\hline 2 & 2 & 2 & 0 & 4 & 0 & 4 & 28.41 & 20.06 & 12.45 & 7.02 & 3.06 & 0.84 & 0.00 \\
\hline 2 & 2 & 1 & 1 & 4 & 0 & 4 & 7.07 & 0.43 & 0.33 & 0.20 & 0.09 & 0.03 & 0.00 \\
\hline 2 & 1 & 2 & 1 & 4 & 0 & 4 & 1.51 & 1.36 & 0.97 & 0.55 & 0.21 & 0.04 & $0 . \infty 0$ \\
\hline 2 & 2 & 2 & 0 & 3 & 1 & 4 & 41.00 & 65.48 & 72.51 & 76.61 & 71.20 & 72.39 & 37.28 \\
\hline 2 & 2 & 1 & 1 & 3 & 1 & 4 & 15.44 & 4.16 & 5.64 & 7.39 & 10.11 & 14.30 & 26.69 \\
\hline 2 & 2 & 0 & 2 & 3 & 1 & 4 & 0.65 & 2.79 & 3.33 & 4.42 & 6.45 & 10.31 & 34.55 \\
\hline
\end{tabular}

In Fig. 2 the deformation density map of the ${ }^{1} \Sigma^{+}$ ground state of the NiGe molecule is shown at the internuclear distance 4.60 a.u. The deformation density has been derived by subtracting the spherical averaged superpositioned atomic charge densities derived in the HF calculations from the molecular charge density determined in the CI calculations. It is recognized that the chemical bond results in a pronounced buildup of charge in the region between the two nuclei.

According to a molecular orbital picture it is expected that the closed shell ${ }^{1} \Sigma^{+}(2 \delta)^{4}(6 \pi)^{4}(12 \sigma)^{2}(13 \sigma)^{2}(14 \sigma)^{2}$ state should be the ground state of the molecule. However, from Table II it is recognized that the energy of this state as derived in the $\mathrm{HF}$ calculations is far above $(\sim 3.58 \mathrm{eV})$ the dissociation limit of the molecule. In Table IV we find the reason why the HF wave function for the closed shell ${ }^{1} \Sigma^{+}$ state does not describe the electronic ground state of the $\mathrm{NiGe}$ molecule. It is due to the fact that the $7 \pi$ orbital which is empty in the closed shell ${ }^{1} \Sigma^{+}$state acquires a population of $0.72 e$ in the $\mathrm{CI}$ wave function. This reveals that the delocalization of the $6 \pi$ orbital that is enforced through the HF calculations on the closed shell ${ }^{1} \Sigma^{+}(2 \delta)^{4}(6 \pi)^{4}(12 \sigma)^{2}(13 \sigma)^{2}(14 \sigma)^{2}$ state is inadequate for describing the ${ }^{1} \Sigma^{+}$electronic ground state of the NiGe molecule. In order to describe a bound molecule, it is necessary to allow for a localized hole in the $3 d \pi$ orbital of $\mathrm{Ni}$, and this is accomplished in the CI calculations through the large population of the $7 \pi$ orbital. In addition, the numbers of $3 d$ and $4 s$ electrons on $\mathrm{Ni}$ in the closed shell ${ }^{1} \Sigma^{+}$state are $9.60 e$ and $0.52 e$, respectively. This indicates that the closed shell ${ }^{1} \Sigma^{+}$ state described in the HF calculations originates from the
$(3 d){ }^{10}$ configuration of the Ni atom. Since the ${ }^{1} S(3 d){ }^{10}$ term of $\mathrm{Ni}$ is located $1.72 \mathrm{eV}$ above the ${ }^{3} D(3 d)^{9}(4 s)^{1}$ ground term, ${ }^{24}$ it is gratifying that the closed shell ${ }^{1} \Sigma^{+}$state does not represent the electronic ground state of the $\mathrm{NiGe}$ molecule.

\section{The low-lying excited electronic states of the NiGe molecule}

Once the nature of the chemical bond in the ${ }^{1} \Sigma^{+}$ground state of the NiGe molecule is understood, it is also understandable that the molecule has quite a few low-lying electronic states. Thus, all these states originate from couplings of the localized holes in the various $3 d$ subshells of $\mathrm{Ni}$ to the localized electron in the $4 p \pi$ orbital of Ge.

The configuration $(3 d \pi)^{3}(4 p \pi)^{1}$ gives rise to the states ${ }^{1} \Sigma^{+},{ }^{1} \Sigma^{-},{ }^{1} \Delta,{ }^{3} \Sigma^{+},{ }^{3} \Sigma^{-}$, and ${ }^{3} \Delta$. From Fig. 1 and Table III it is recognized that states of these symmetries are present within the energy range $0.7 \mathrm{eV}$, and a closer examination of their wave functions reveal that they indeed originate from the configuration $(3 d \pi)^{3}(4 p \pi)^{1}$.

The electronic states with transition energies ranging from 0.34 to $0.37 \mathrm{eV}$ are exactly those derivable from the configuration $(3 d \delta)^{3}(4 p \pi)^{1}$, namely ${ }^{1} \Phi,{ }^{3} \Pi,{ }^{3} \Phi$, and ${ }^{1} \Pi$. The ${ }^{1} \Pi$ and ${ }^{3} \Pi$ states with transition energies 0.57 and 0.60 $\mathrm{eV}$, respectively, are due to the configuration $(3 d \sigma)^{1}(4 p \pi)^{1}$.

Thus, all the electronic states with transition energies below or equal to $0.66 \mathrm{eV}$ are due to the interaction between the Ge atom in the $(4 p \sigma)^{1}(4 p \pi)^{1}$ valence shell configuration and the $\mathrm{Ni}$ atom in the $(3 d)^{9}(4 s)^{1}$ configuration with the hole in the $\mathrm{Ni} 3 d$ shell being localized either in the $3 d \delta$, the $3 d \pi$, or the $3 d \sigma$ subshell. In all these states the delocalized

TABLE VI. Occupation of the natural valence orbitals in the ' $\Sigma+$ ground state of the NiGe molecule as functions of the internuclear distance.

\begin{tabular}{lcccccccc}
\hline \hline & \multicolumn{7}{c}{ Occupation } \\
\cline { 2 - 8 } Orbital & 3.6 & 4.2 & 4.6 & 5.0 & 5.5 & 6.2 & 12.0 \\
\hline $12 \sigma(\sim \mathrm{Ge} 4 s \sigma)$ & 1.99 & 1.98 & 1.98 & 1.97 & 1.97 & 1.97 & 1.97 \\
$13 \sigma(\sim \mathrm{Ni3} d \sigma)$ & 1.98 & 1.99 & 1.99 & 1.99 & 2.00 & 2.00 & 2.00 \\
$14 \sigma(\sim \mathrm{Ni4} s+\mathrm{Ge}+p \sigma)$ & 1.88 & 1.88 & 1.87 & 1.84 & 1.78 & 1.67 & 1.03 \\
$15 \sigma(\sim \mathrm{Ni4} s-\mathrm{Ge} 4 p \sigma)$ & 0.13 & 0.13 & 0.14 & 0.16 & 0.22 & 0.34 & 0.97 \\
$6 \pi(\sim \mathrm{Ni3} d \pi)$ & 3.58 & 3.43 & 3.22 & 3.21 & 3.10 & 3.03 & 3.00 \\
$7 \pi(\sim \mathrm{Ge} 4 p \pi)$ & 0.45 & 0.60 & 0.72 & 0.83 & 0.93 & 1.00 & 1.03 \\
$2 \delta(\sim \mathrm{Ni} 3 d \delta)$ & 3.99 & 3.99 & 3.99 & 4.00 & 4.00 & 4.00 & 4.00 \\
\hline \hline
\end{tabular}




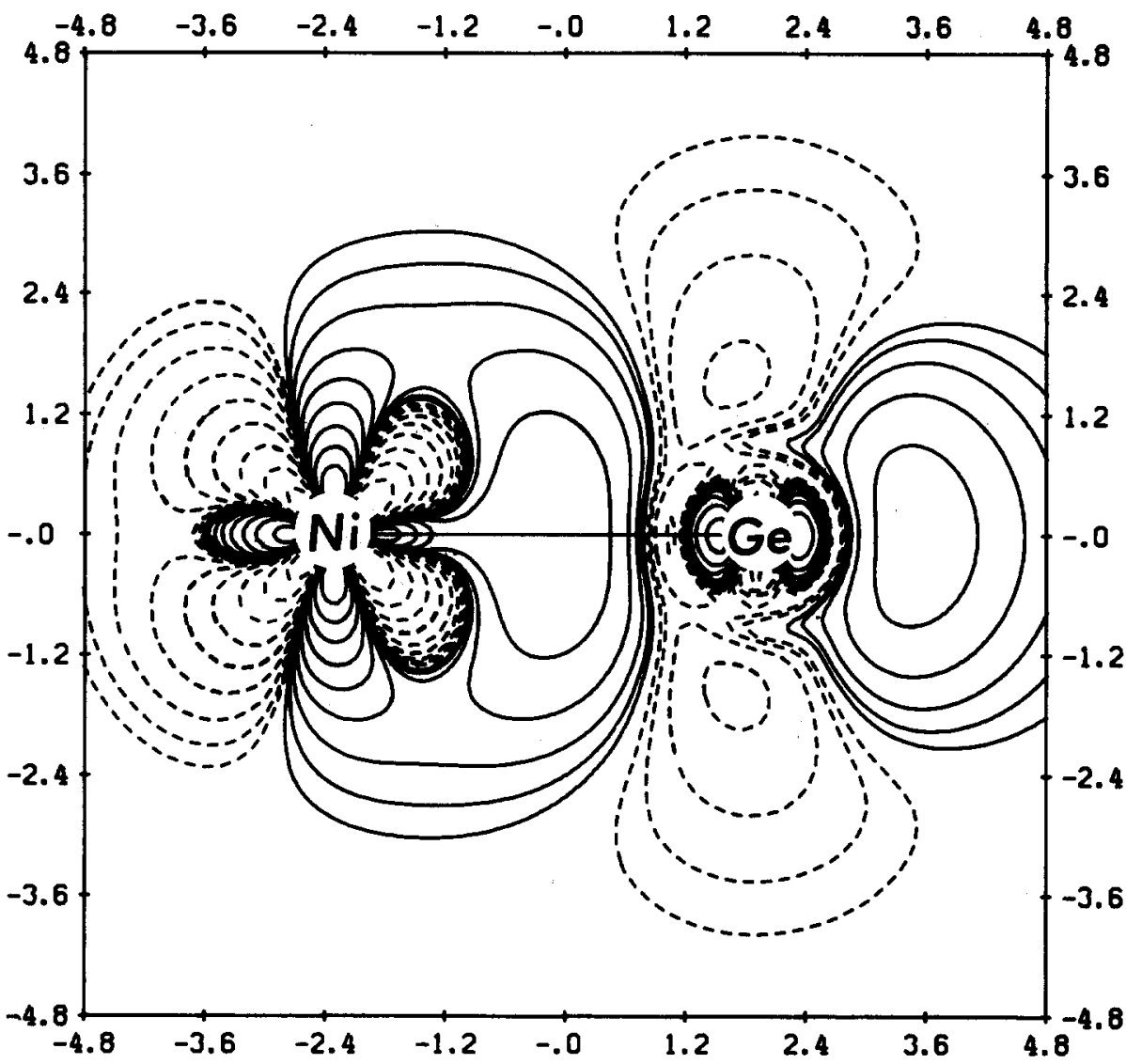

FIG. 2. Deformation density map for the ${ }^{1} \Sigma^{+}$ground state of the NiGe molecule at the internuclear distance 4.60 a.u. The superpositioned atomic charge densities from the $(3 d)^{9}(4 s)^{1}$ configuration of $\mathrm{Ni}$ and the $(4 s)^{2}(4 p)^{2}$ configuration of $\mathrm{Ge}$ has been subtracted from the molecular charge density. Solid ourves show enhanced electron charge relative to the superpositioned atoms, dashed contours show diminished charge. The smallest contour value is 0.000625 e/a.u. ${ }^{3}$ Adjacent contours differ by a factor of 2 .

molecular orbital composed of the $4 s$ orbital of $\mathrm{Ni}$ and the $4 p \sigma$ orbital of Ge is doubly occupied at the equilibrium distance of the molecule.

At this point it is interesting to compare the results obtained in the present work with our previous results on the molecules $\mathrm{Ni}_{2},{ }^{3,11} \mathrm{Ge}_{2}$, ${ }^{12,13}$ and PdGe. ${ }^{9}$

The $\mathrm{Ge}_{2}$ molecule has a ${ }^{3} \Sigma_{g}^{-}$ground state with the configuration $\left(4 p \sigma_{g}\right)^{2}\left(4 p \pi_{u}\right)^{2}$ and a low-lying excited state ${ }^{3} \Pi_{u}\left(4 p \sigma_{g}\right)^{1}\left(4 p \pi_{u}\right)^{3}$. This is consistent with our findings that the lowest-lying electronic states of the NiGe molecule originates from the $(4 p \sigma)^{1}(4 p \pi)^{1}$ configuration of $\mathrm{Ge}$ and that low-lying electronic states attributed to the $(4 p \pi)^{2}$ configuration are also present as discussed below.

As part of the $\mathrm{Ni}_{2}$ molecule each $\mathrm{Ni}$ atom has a localized hole in the $3 d$ shell. The lowest-lying states in $\mathrm{Ni}_{2}$ are those arising from the valence bond couplings between the localized $3 d \delta$ holes on each $\mathrm{Ni}$ atom. The $3 d \pi$ hole states are located approximately in the middle of the "band" consisting of the 30 low-lying electronic states, and the highestlying states in this band are the $3 d \sigma$ hole states. 3,11

As part of the NiGe molecule it is noted that the states arising from the $3 d \pi$ holes on Ni, i.e., ${ }^{1} \Sigma^{+},{ }^{3} \Sigma^{+},{ }^{3} \Delta,{ }^{3} \Sigma^{-},{ }^{1} \Delta$, and ${ }^{1} \Sigma^{-}$, are having transition energies ranging from 0.00 to $0.66 \mathrm{eV}$. Contrary to this, the states originating from the $3 d \delta$ holes on $\mathrm{Ni},{ }^{1} \Phi,{ }^{3} \Pi,{ }^{3} \Phi$, and ${ }^{1} \Pi$, are located close together with transition energies between 0.34 and $0.37 \mathrm{eV}$, and those due to the $3 d \sigma$ holes on $\mathrm{Ni},{ }^{1} \Pi$ and ${ }^{3} \Pi$, have the transition energies 0.57 and $0.60 \mathrm{eV}$, respectively.

The energy differences between the $3 d \delta$ hole states and the $3 d \pi$ hole states ${ }^{3} \Sigma^{-},{ }^{1} \Delta$, and ${ }^{1} \Sigma^{-}$are in agreement with our findings for the $\mathrm{Ni}_{2}$ molecule. Therefore, we conclude that the remaining $3 d \pi$ hole states, ${ }^{1} \Sigma^{+},{ }^{3} \Sigma^{+}$, and ${ }^{3} \Delta$ as well as the $3 d \sigma$ hole states, ${ }^{3} \Pi$ and ${ }^{1} \Pi$, get lowered in energy due to the interactions between the $3 d \pi$ or $3 d \sigma$ hole on $\mathrm{Ni}$ and the valence orbitals, $4 p \pi$ or $4 p \sigma$, of the Ge atom.

Comparison with our previous $a b$ initio results for the PdGe molecule reveals that the nature of the chemical bond as well as the spectra of the low-lying electronic states of the $\mathrm{NiGe}$ molecule differ conspicuously from those of the PdGe molecule. However, this is due to the fact that the ground terms of the transition metal atoms arise from different orbital configurations, i.e., $(4 d)^{10}$ for $\mathrm{Pd}$ and $(3 d)^{9}(4 s)^{1}$ for $\mathrm{Ni}$.

The five highest-lying states in Fig. 1. and Table III, ${ }^{5} \Delta$, ${ }^{3} \Delta,{ }^{5} \Pi,{ }^{3} \Pi$, and ${ }^{5} \Sigma^{-}$, all arise from the interaction between the $\mathrm{Ni}$ atom in the $(3 d)^{9}(4 s)^{1}$ configuration and the $\mathrm{Ge}$ atom in the $(4 p \pi)^{2}$ configuration. The states ${ }^{5} \Delta$ and ${ }^{3} \Delta$ each have a localized hole in the $3 d \delta$ subshell of $\mathrm{Ni}$, the ${ }^{5} \Pi$ and ${ }^{3} \Pi$ states have a localized hole in the $\mathrm{Ni} 3 d \pi$ subshell whereas the localized $3 d \sigma$ hole of $\mathrm{Ni}$ gives rise to the ${ }^{5} \Sigma^{-}$state as well as a ${ }^{3} \Sigma^{-}$state. The latter has a somewhat higher transition energy, namely $1.16 \mathrm{eV}$, than the remaining five states, and it is therefore not included in Fig. 1 or Table III.

It is recognized that the abovementioned six states all arise from the ${ }^{3} \Sigma-$ coupling of the $(4 p \pi)^{2}$ valence shell configuration of the Ge atom. The ${ }^{3} \Sigma^{-}$valence shell coupled electrons of $\mathrm{Ge}$ thereafter couple to the $3 d \delta$ hole of $\mathrm{Ni}$ yielding the ${ }^{4} \Delta$ and ${ }^{2} \Delta$ states, to the $3 d \pi$ hole of Ni yielding the ${ }^{4} \Pi$ and ${ }^{2} \Pi$ states, and finally to the $3 d \sigma$ hole of Ni yielding ${ }^{4} \Sigma^{-}$ and ${ }^{2} \Sigma-$ states. The final states of the NiGe molecule, ${ }^{5} \Delta,{ }^{3} \Delta$, ${ }^{5} \Pi,{ }^{3} \Pi,{ }^{5} \Sigma^{-}$, and ${ }^{3} \Sigma^{-}$, are derived by high spin couplings of 
the quartet and doublet states with a singly occupied $\sigma$ orbital which is mostly the $\mathrm{Ni} 4 s$ orbital.

Although the singly occupied $\sigma$ orbital in the highest lying states in Fig. 1 and Table III is mostly the $\mathrm{Ni} 4 s$ orbital, it does mix with the empty $4 p \sigma$ orbital of Ge. This causes a significant charge transfer from the $\mathrm{Ni}$ to the $\mathrm{Ge}$ atoms resulting in a rather large negative net gross atomic charge on Ge. The singly occupied $\sigma$ orbital is essentially the delocalized molecular orbital that plays an important role for the formation of the chemical bond in the ground state of the $\mathrm{NiGe}$ molecule. Therefore it is intuitively clear that the electronic states arising from the $(4 p \pi)^{2}$ configuration of $\mathrm{Ge}$ are all located at higher energies than those arising from the $(4 p \sigma)^{1}(4 p \pi)^{1}$ valence shell configuration of the Ge atom.

Finally it is noted that the states arising from the interaction between the $(3 d)^{9}(4 s)^{1} \mathrm{Ni}$ atom and the $(4 p \pi)^{2} \mathrm{Ge}$ atom, ${ }^{5} \Delta,{ }^{3} \Delta,{ }^{5} \Pi,{ }^{3} \Pi$, and ${ }^{5} \Sigma^{-}$, can be arranged energetically according to the symmetry of the $3 d$ hole on Ni. Thus, the $3 d \delta$ hole states are located at lower energies than the $3 d \pi$ hole states, and the $3 d \pi$ hole states at lower energy than the $3 d \sigma$ hole states. This finding is in accordance with our results for the $\mathrm{Ni}_{2}$ molecule. ${ }^{3,11}$

\section{MASS SPECTROMETRIC MEASUREMENTS}

A $90^{\circ}$ sector, 12 in. radius, magnetic focusing type mass spectrometer equipped with a Knudsen cell assembly was employed for measuring the equilibrium vapor composition. A tantalum Knudsen cell and the inner graphite cell had 0.040 in. diameter concentric orifices with 0.02 in. in length each. The graphite cell was charged with $111 \mathrm{mg}$ of germanium powder, $69 \mathrm{mg}$ of nickel powder and $18 \mathrm{mg}$ of silver wire (as calibrant). Heating was effected by radiation from a thoriated tungsten resistance heater surrounding the Knudsen cell. The temperatures were measured by sighting on a blackbody hole at the base of the cell. The optical pyrometer used had previously been calibrated in situ at the melting point of an NBS reference gold sample, inside a graphite cell.

The molecular beam effusing from the sample cell into the ion source region of the mass spectrometer was ionized with $20 \mathrm{~V}$ electrons. Other operational conditions were an emission current of $0.40 \mathrm{~mA}$, a filament current of $6.20 \mathrm{~A}$, a $4.5 \mathrm{kV}$ acceleration potential and a $0.08 \mathrm{~mA}$ dynode string current. The detector was a 20-stage electron multiplier, whose entrance shield was maintained at $\sim-2 \mathrm{kV}$ potential. Before reaching the electron multiplier, the ions passed through a 50\% transmission grid collector so that the multiplier current gain could be determined readily for the most intense species.

The parent molecules were identified by their mass-tocharge ratio, isotopic abundancies, shutter effect, and ionization efficiency curves. The total ion currents for the pertinent species discussed in this paper are listed in Table VII.

Several data sets of $\mathrm{Ag}^{+}$and $\mathrm{Ag}_{2}^{+}$measured at the lowest temperatures were used to determine the instrument constant $k_{\mathrm{Ag}}$ by evaluating the equilbrium reaction $\mathrm{Ag}_{2}(\mathrm{~g})=2 \mathrm{Ag}(\mathrm{g})$. The necessary enthalpy $\mathrm{D}_{0}^{\circ}\left(\mathrm{Ag}_{2}\right)$ $=172.0 \pm 6.3 \mathrm{~kJ} \mathrm{~mol}^{-1}$ and the Gibbs energy functions were taken from Ref. 15. The corresponding $k_{i}$ values for the other species were calculated by the relationship

TABLE VII. Measured total ion intensities of the pertinent species containing nickel and germanium."

\begin{tabular}{|c|c|c|c|c|}
\hline \multirow[b]{2}{*}{ Temp (K) } & \multicolumn{4}{|c|}{ Ion Intensities (in A) } \\
\hline & $\mathrm{Ge}^{+}$ & $\mathrm{Ge}_{2}^{+}$ & $\mathbf{N i}^{+}$ & $\mathrm{NiGe}^{+}$ \\
\hline 1719 & $3.176(-8)$ & $8.437(-11)$ & $2.983(-9)$ & $3.470(-12)$ \\
\hline 1701 & $2.683(-8)$ & $5.301(-11)$ & $2.369(-9)$ & $2.248(-12)$ \\
\hline 1621 & $9.054(-9)$ & $1.776(-11)$ & $6.014(-10)$ & $4.688(-13)$ \\
\hline 1675 & $1.783(-8)$ & $3.274(-11)$ & $1.196(-9)$ & $9.400(-13)$ \\
\hline 1709 & $3.446(-8)$ & $8.395(-11)$ & $3.007(-9)$ & $3.062(-12)$ \\
\hline 1750 & $5.064(-8)$ & $1.448(-10)$ & $4.906(-9)$ & $4.976(-12)$ \\
\hline 1802 & $9.278(-8)$ & $3.203(-10)$ & $9.797(-9)$ & $1.440(-11)$ \\
\hline 1840 & $1.492(-7)$ & $5.503(-10)$ & $1.940(-8)$ & $3.028(-11)$ \\
\hline 1874 & $2.052(-7)$ & $8.298(-10)$ & $2.846(-8)$ & $4.588(-11)$ \\
\hline 1825 & $1.040(-7)$ & $3.778(-10)$ & $1.445(-8)$ & $1.376(-11)$ \\
\hline 1784 & $8.042(-8)$ & $2.350(-10)$ & $9.664(-9)$ & $1.193(-11)$ \\
\hline 1720 & $3.003(-8)$ & $6.712(-11)$ & $3.129(-9)$ & $2.768(-12)$ \\
\hline 1750 & $4.841(-8)$ & $1.028(-10)$ & $5.171(-9)$ & $5.016(-12)$ \\
\hline 1798 & $1.004(-7)$ & $2.808(-10)$ & $1.190(-8)$ & $1.063(-11)$ \\
\hline 1839 & $1.488(-7)$ & $5.629(-10)$ & $2.326(-8)$ & $3.193(-11)$ \\
\hline 1886 & $2.402(-7)$ & $9.155(-10)$ & $4.508(-8)$ & $6.148(-11)$ \\
\hline 1913 & $3.170(-7)$ & $1.171(-9)$ & $7.251(-8)$ & $9.482(-11)$ \\
\hline 1948 & $4.289(-7)$ & $1.545(-9)$ & $1.136(-7)$ & $1.569(-10)$ \\
\hline 1904 & $2.510(-7)$ & $7.404(-10)$ & $6.447(-8)$ & $6.698(-11)$ \\
\hline 1857 & $1.736(-7)$ & $4.495(-10)$ & $4.552(-8)$ & $4.074(-11)$ \\
\hline 1854 & $7.549(-8)$ & $1.070(-10)$ & $1.883(-8)$ & $7.646(-12)$ \\
\hline 1876 & $1.906(-7)$ & $4.886(-10)$ & $5.187(-8)$ & $5.056(-11)$ \\
\hline 1916 & $3.009(-7)$ & $9.218(-10)$ & $9.817(-8)$ & $1.058(-10)$ \\
\hline 1941 & $3.936(-7)$ & $1.263(-9)$ & $1.456(-7)$ & $1.633(-10)$ \\
\hline 1961 & $4.896(-7)$ & $1.456(-9)$ & $1.843(-7)$ & $2.199(-10)$ \\
\hline 2003 & $6.216(-7)$ & $1.684(-9)$ & $2.842(-7)$ & $3.038(-10)$ \\
\hline
\end{tabular}

Numbers in parentheses represent powers of ten. 
$k_{i}=k_{\mathrm{Ag}} \sigma_{\mathrm{Ag}} \gamma_{\mathrm{Ag}} E_{i} / \sigma_{i} \gamma_{i} E_{\mathrm{Ag}}$. The relative maximum atomic ionization cross sections $\sigma_{i}$ were taken from $\operatorname{Mann}^{25}$ for the atoms. The ionization cross sections for the molecular species were estimated as 0.75 times the sum of the atomic cross sections. ${ }^{26}$ The multiplier gains $\gamma_{i}$ were experimentally determined for the species $\mathrm{Ag}, \mathrm{Ge}$, and $\mathrm{Ni}$ and estimated for the molecules. The factors $E_{i}$, which correct the measured ion intensities to the maximum ionization efficiencies were obtained from the experimental ionization efficiency curves. All the measured and estimated instrument parameters are listed in Table VIII. The appearance potentials listed in Table VIII were determined by the linear extrapolation method using that for $\mathrm{Ag}$ equal of $7.574 \mathrm{eV}$ (27) as reference.

The Gibbs energy functions, $-\left(G_{T}^{\circ}-H_{298}^{\circ}\right) / T$, and the heat content functions were taken from Hultgren et al. ${ }^{28}$ for atomic nickel and germanium and from Kingcade et al. ${ }^{12}$ for the gaseous molecule $\mathrm{Ge}_{2}$. For $\mathrm{NiGe}(\mathrm{g})$ the thermal functions were computed according to statistical thermodynamic procedures using the harmonic oscillator, rigid rotator approximation, ${ }^{29}$ and the molecular constants derived in the present theoretical investigation. The bond distance used was $2.28 \AA$, and the vibrational frequency used was 239 $\mathrm{cm}^{-1}$. The electronic contribution employed was due to the calculated energy levels below $4000 \mathrm{~cm}^{-1}$. The derived Gibbs energy functions, $-\left(G_{T}^{\circ}-H_{0}^{\circ}\right) / T$ and enthalpy increments, $H_{T}^{\circ}-H_{0}^{\circ}$ are listed in Table IX.

The dissociation energy of $\mathrm{NiGe}(\mathrm{g})$ was obtained from the experimental equilibrium constants $K_{p}$ for the gas-phase reactions

$$
\begin{aligned}
& \mathrm{NiGe}=\mathrm{Ni}+\mathrm{Ge}, \\
& \mathrm{NiGe}+\mathrm{Ge}=\mathrm{Ni}+\mathrm{Ge}_{2} .
\end{aligned}
$$

The enthalpy change, $\Delta H_{0}^{\circ}$, for reaction (1) was calculated by the third-law relationship, $\Delta H_{0}^{\circ}=-R T \ln K_{p}-T$ $\Delta\left[\left(G_{T}^{\circ}-H_{0}^{\circ}\right) / T\right]$. This yields an enthalpy of $280.0 \pm 2.5$ $\mathrm{kJ} \mathrm{mol}^{-1}$. The same calculation for reaction (2), yielded an enthalpy of $17.1 \pm 2.1 \mathrm{~kJ} \mathrm{~mol}^{-1}$.

The reaction enthalpies were also evaluated from a least square analysis of a plot of $\log K_{p}$ vs $1 / T$. This evaluation yielded an enthalpy change at the average temperature of $1792 \mathrm{~K}$ for both reactions over the range $1621-2003 \mathrm{~K}$. The average second-law enthalpy for reaction (1) was calculated as $\Delta H_{T}^{\circ}=294.0 \pm 9.2 \mathrm{~kJ} \mathrm{~mol}^{-1}$, and for reaction (2) as $14.8 \pm 7.1 \mathrm{~kJ} \mathrm{~mol}^{-1}$. The corresponding enthalpies referenced to $0 \mathrm{~K}$ are $\Delta H_{0}^{\circ}=291.2 \mathrm{~kJ} \mathrm{~mol}^{-1}$ for reaction (1) and $\Delta H_{0}^{\circ}=38.1 \mathrm{~kJ} \mathrm{~mol}^{-1}$ for reaction (2). The above reac-
TABLE IX. Gibbs energy function, $-\left(G_{T}^{\circ}-H_{0}^{\circ}\right) / T$ in $\mathrm{J} \mathrm{K}^{-1} \mathrm{~mol}^{-1}$, and heat content function, $H_{T}^{\circ}-H_{0}^{\circ}$ in $\mathrm{kJ} \mathrm{mol}^{-1}$, for the gaseous molecule NiGe.

\begin{tabular}{ccc}
\hline$T(\mathrm{~K})$ & $-\left(G_{T}^{\circ}-H_{0}^{\circ}\right) / T$ & $H_{T}^{\circ}-H_{0}^{\circ}$ \\
\hline 298.15 & 215.4 & 9.995 \\
1300 & 271.5 & 61.01 \\
1400 & 275.0 & 66.65 \\
1500 & 278.3 & 72.04 \\
1600 & 281.4 & 77.28 \\
1700 & 284.4 & 82.30 \\
1800 & 287.1 & 87.15 \\
1900 & 289.8 & 91.85 \\
2000 & 292.2 & 96.43 \\
2100 & 294.6 & 100.9 \\
\hline
\end{tabular}

tion enthalpies are summarized in Table $X$, along with the evaluated entropies.

The dissociation energy $D_{0}^{\circ}$ for $\mathrm{NiGe}(\mathrm{g})$ was calculated at $285.6 \pm 9.2\left(D_{298}^{\circ}=289.9\right) \mathrm{kJ} \mathrm{mol}^{-1}$, from the average of the second- and third-law enthalpies of reaction (1). Reaction (1) yields directly the dissociation energy of $\mathrm{NiGe}(\mathrm{g})$. A dissociation energy of $D_{0}^{\circ}=288.0 \pm 14.3$ $\left(D_{298}^{\circ}=290.8\right) \mathrm{kJ} \mathrm{mol}^{-1}$ was obtained from the average of the second- and third-law enthalpies for reaction (2), by using the literature value of $D_{0}^{\circ} \quad\left(\mathrm{Ge}_{2}\right)=260.4 \pm 7.0$ $\left(D_{298}^{\circ}=263.5\right) \mathrm{kJ} \mathrm{mol}^{-1} \cdot{ }^{12}$ The final selected dissociation energy of $\mathrm{NiGe}(\mathrm{g})$ is taken as the average of these two calculated dissociation energies and is $D_{0}^{\circ}=286.8 \pm 10.9$ $\left(D_{298}^{\circ}=290.3\right) \mathrm{kJ} \mathrm{mol}^{-1}$. The error term represents an estimated error of the average value.

This selected value for the dissociation energy of $\mathrm{NiGe}$ (g) compares favorably with the reported literature value by Kant, ${ }^{14}$ of $D_{0}^{\circ}=277.8 \pm 16.7 \mathrm{~kJ} \mathrm{~mol}^{-1}$. The discrepancy in the two evaluated energies seems to be in the electronic contribution to the thermal functions. Miedema and Gingerich ${ }^{30}$ have derived the dissociation energy of $\mathrm{NiGe}$ as $296 \mathrm{~kJ} \mathrm{~mol}^{-1}$ using their atomic cell model.

The corresponding enthalpy of formation for the gaseous molecule $\mathrm{NiGe}$ is obtained as $\Delta H_{f, 0}^{\circ}=513 \pm 12$ $\left(\Delta H_{f, 298}^{\circ}=514\right) \mathrm{kJ} \mathrm{mol}^{-1}$. This heat of formation was evaluated employing the value of Ge vaporization as $371.7 \pm 2.1$ $\mathrm{kJ} \mathrm{mol}{ }^{-1}$ and $\mathrm{Ni}$ vaporization as $428.1 \pm 2.1 \mathrm{~kJ} \mathrm{~mol}^{-1}$, which were taken from Hultgren $e t a l .^{26}$ and referenced to 0 K.

TABLE VIII. Experimental and estimated instrument parameters used in the evaluation of $k_{i}$.

\begin{tabular}{lccccc}
\hline \hline Ion & $\begin{array}{c}\text { Appearance } \\
\text { potential (eV) }\end{array}$ & $\begin{array}{c}\text { Relative } \\
\text { ionization } \\
\text { cross section }\end{array}$ & $\begin{array}{c}\text { Multiplier } \\
\text { gain }\end{array}$ & $\begin{array}{c}I^{+} \\
\text {Correction } \\
\text { factor }\left(E_{i}\right)\end{array}$ & $\begin{array}{c}\left.k_{i} \times 10^{-3}\right) \\
\left(\text { atm } / \mathrm{A}^{-1} \mathbf{K}^{-1}\right)\end{array}$ \\
\hline $\mathrm{Ag}$ & $7.6 \pm 0.3$ & 5.05 & 2.53 & 1.03 & $14.00 \pm 4.30$ \\
$\mathrm{Ni}$ & $8.7 \pm 0.4$ & 7.58 & 3.64 & 1.02 & 9.46 \\
$\mathrm{NiGe}$ & $6.9 \pm 0.5$ & 8.39 & $(3.24)$ & 1.05 & 6.80 \\
$\mathrm{Ge}$ & $9.3 \pm 0.4$ & 5.71 & 2.68 & 1.03 & 12.20 \\
$\mathrm{Ge}$ & $9.6 \pm 0.5$ & 8.56 & $(2.68)$ & 1.01 & 7.75 \\
\hline
\end{tabular}

Numbers in parentheses are estimated values. 
TABLE X. Summary of reaction enthalpies, in $\mathrm{kJ} \mathrm{mol}^{-1}$, and reaction entropies, in $\mathrm{J} \mathrm{K}^{-1} \mathrm{~mol}^{-1}$, for the molecule $\mathrm{NiGe}(\mathrm{g})$.

\begin{tabular}{|c|c|c|c|c|c|c|c|}
\hline \multirow[b]{2}{*}{ Reaction } & \multirow[b]{2}{*}{$\begin{array}{c}\Delta H_{T}^{\circ} \\
\text { Second law }\end{array}$} & \multicolumn{2}{|c|}{$\Delta H_{0}^{\circ}$} & \multirow[b]{2}{*}{$\begin{array}{c}\Delta S_{r}^{\circ} \\
\text { Second law }\end{array}$} & \multicolumn{2}{|c|}{$\Delta S_{298}^{0}$} & \multirow{2}{*}{$\begin{array}{c}\text { Av. } \\
D_{0}^{\circ} \\
\mathrm{NiGe}\end{array}$} \\
\hline & & $\begin{array}{l}\text { Second } \\
\text { law }\end{array}$ & $\begin{array}{l}\text { Third } \\
\text { law }\end{array}$ & & $\begin{array}{l}\text { Second } \\
\text { law }\end{array}$ & $\begin{array}{l}\text { Third } \\
\text { law }\end{array}$ & \\
\hline \multirow[t]{2}{*}{$\begin{array}{c}\mathrm{NiGe}=\mathrm{Ni}+\mathrm{Ge} \\
\mathrm{NiGe}+\mathrm{Ge}=\mathrm{Ni}+\mathrm{Ge}_{2}\end{array}$} & $\begin{array}{r}294.0 \pm 9.2 \\
14.8 \pm 7.1\end{array}$ & $\begin{array}{r}291.2 \pm 9.2 \\
38.1 \pm 7.1\end{array}$ & $\begin{array}{r}280.0 \pm 2.5 \\
17.1 \pm 2.1\end{array}$ & $\begin{array}{r}113.7 \pm 5.0 \\
15.2 \pm 3.9\end{array}$ & $\begin{array}{r}107.0 \pm 5.0 \\
40.1 \pm 3.9\end{array}$ & $\begin{array}{r}100.9 \\
28.7\end{array}$ & $\begin{array}{l}285.6 \pm 9.2 \\
288.0 \pm 14.3\end{array}$ \\
\hline & & & & & & & $286.0 \pm 10.9$ \\
\hline
\end{tabular}

\section{CONCLUSIONS}

In the present work, we have presented results of both theoretical and experimental investigations of the gaseous $\mathrm{NiGe}$ molecule. The electronic structure and the nature of bonding of the molecule has been elucidated through all electron $a b$ initio HF-CI calculations. The molecular constants, i.e., the equilibrium distance and the vibrational frequency obtained for the ${ }^{1} \Sigma^{+}$ground state have been utilized together with the calculated low-lying electronic states to derive the Gibbs energy functions for the NiGe molecule needed to determine the dissociation energy from the mass spectrometric equilibrium data.

The experimental dissociation energy of the NiGe molecule as determined in the present work is $D_{0}^{\circ}=286.8 \pm 10.9$ $\mathrm{kJ} \mathrm{mol}^{-1}$.

Our ab initio work is especially concerned with the interaction between the ground term $\mathrm{Ni}$ and $\mathrm{Ge}$ atoms, i.e., $\mathrm{Ni}$ ${ }^{3} D(3 d)^{9}(4 s)^{1}$ and $\mathrm{Ge}^{3} P(4 s)^{2}(4 p)^{2}$. Based on the results of the CI calculations, the $\mathrm{NiGe}$ molecule is predicted to have a ${ }^{1} \Sigma^{+}$ground state. However, we also predict that the NiGe molecule has numerous low-lying electronic states due to a localized hole in either the $3 d \delta$, the $3 d \pi$, or the $3 d \sigma$ subshell of the $\mathrm{Ni}$ atom.

The chemical bond in the ${ }^{1} \Sigma^{+}$ground state is a double bond composed of one $\sigma$ bond and one $\pi$ bond. The $\sigma$ bond is due to the doubly occupied delocalized molecular orbital formed of the Ni $4 s$ orbital and the Ge $4 p \sigma$ orbitals. The $\pi$ bond primarily arises from the valence bond coupling of the $3 d \pi$ hole on $\mathrm{Ni}$ and the $4 p \pi$ electron on $\mathrm{Ge}$, although some delocalization occurs.

\section{ACKNOWLEDGMENTS}

The computations have been performed at the Computing Service Center at Texas A\&M University and at UNI $\cdot C$, the Technical University of Denmark. The work at Texas A\&M University has been supported by the Welch Foundation and the National Science Foundation. The authors (I.S. and K.A.G.) appreciate the support by a NATO Grant No. RG. 85/0448 for international collaboration in research. I.S. acknowledges the Royal Danish Academy of Sciences and Letters for awarding the Niels Bohr fellowship.
'Faraday Symposia of the Royal Society of Chemistry No. 14, Diatomic Metals and Metallic Clusters (1980).

${ }^{2}$ Metal Bonding and Interactions in High Temperature Systems, edited by J. L. Gole and W. C. Stwalley, American Chemical Society Symposium Ser. 178 (American Chemical Society, Washington, D.C., 1982).

${ }^{3}$ I. Shim, Kgl. Danske Vid. Selsk. Matt.-Fys. Medd. 41, 147 (1985).

${ }^{4}$ M. D. Morse, Chem. Rev. 86, 1049 (1986).

${ }^{5}$ I. Shim and K. A. Gingerich, J. Chem. Phys. 76, 3833 (1982).

${ }^{6}$ I. Shim and K. A. Gingerich, J. Chem. Phys. 81, 5937 (1984).

${ }^{7}$ I. Shim and K. A. Gingerich, Surf. Sci. 156, 623 (1985).

${ }^{8}$ I. Shim, H. C. Finkbeiner, and K. A. Gingerich, J. Phys. Chem. 91, 3171 (1987).

${ }^{9}$ I. Shim, J. E. Kingcade, Jr., and K. A. Gingerich, J. Chem. Phys. 85, 6629 (1986).

${ }^{10}$ I. Shim, J. E. Kingcade, Jr., and K. A. Gingerich, Z. Phys. D 7, 261 (1987).

"I. Shim, J. P. Dahl, and H. Johansen, Int. J. Quantum Chem. 15, 311 (1979).

${ }^{12}$ J. E. Kingcade, Jr., H. M. Nagarathna-Naik, I. Shim, and K. A. Gingerich, J. Phys. Chem. 90, 2830 (1986).

${ }^{13}$ I. Shim, H. M. Nagarathna-Naik, and K. A. Gingerich, Int. J. Quantum Chem. 29, 975 (1986).

${ }^{14}$ A. Kant, J. Chem. Phys. 44, 2450 (1966).

${ }^{15} \mathrm{~J}$. E. Kingcade, Ph.D. dissertation, Texas A\&M University, 1983.

${ }^{16}$ C. C. J. Roothaan, Rev. Mod. Phys. 32, 179 (1960).

${ }^{17} \mathrm{~J}$. Almlof, in Proceedings of the Second Seminar on Computational Problems in Quantum Chemistry (Max Planck Institut, Munchen, 1973).

${ }^{18}$ The ALCHEMY program system has been written at IBM Research Laboratory in San Jose, CA, by P. S. Bagus, B. Liu, M. Yoshimine, and A. D. McLean.

${ }^{19}$ C. R. Sarma and S. Rettrup, Theor. Chim. Acta (Berlin) 46, 63 (1977); S. Rettrup and C. R. Sarma, ibid. 46, 73 (1977).

${ }^{20} \mathrm{H}$. Johansen (private communication).

${ }^{21}$ A. J. H. Wachters, J. Chem. Phys. 52, 1033 (1970).

${ }^{22}$ S. Huzinaga, J. Chem. Phys. 66, 4245 (1977).

${ }^{23}$ C. R. Claydon and K. D. Carlson, J. Chem. Phys. 49, 1331 (1968).

${ }^{24}$ C. E. Moore, Natl. Bur. Stand. Circ. No. 467 (U. S. GPO, Washington, D.C., 1952), Vol. 2.

${ }^{25}$ J. B. Mann, in Recent Developments in Mass Spectrometry, Proceedings of the International Conference on Mass Spectrometry, edited by K. Ogata and T. Hayakawa (University of Tokyo, Tokyo, 1970), pp. 814-819.

${ }^{26} \mathrm{~J}$. Drowart and P. Goldfinger, Angew. Chem. Int. Ed. Engl. 6, 581 (1967).

${ }^{27}$ C. E. Moore, Natl. Bur. Stand. Circ. No. 467 (U. S. GPO, Washington, D.C., 1958), Vol. 3.

${ }^{28}$ R. Hultgren, P. J. Desai, D. T. Hawkins, M. Gleiser, K. K. Kelly, and D. D. Wagman, Selected Values of Thermodynamic Properties of the Elements (American Society for Metals, Metal Park, OH, 1973).

${ }^{29} \mathrm{D}$. R. Stull and H. Prophet, in The Characterization of High Temperature Vapors, edited by J. L. Margrave (Wiley-Interscience, New York, 1967), pp. 359-424.

${ }^{30}$ A. R. Miedema and K. A. Gingerich, J. Phys. B 12, 2081, 2255 (1979). 